Original Research Article

\title{
Study of prescription pattern in a tertiary care hospital in Chhattisgarh, India: an observational study
}

\author{
Purnima Raj, Subhankar Choudhury*, Sumati Kundu, Satyam Patel, Bhagwati Sidar
}

\begin{abstract}
Department of Pharmacology, Late Shri Lakhiram Agrawal Memorial Government Medical College, Raigarh, Chhatisgarh, India
\end{abstract}

Received: 20 February 2018 Accepted: 26 February 2018

\section{*Correspondence to: \\ Dr. Subhankar Choudhury, Email: dr.subhankar1985@ gmail.com}

Copyright: (C) the author(s), publisher and licensee Medip Academy. This is an openaccess article distributed under the terms of the Creative Commons Attribution NonCommercial License, which permits unrestricted noncommercial use, distribution, and reproduction in any medium, provided the original work is properly cited.

\begin{abstract}
Background: Prescription audit is one of the methods to assess the drug utilization pattern and rational use of drugs. In our country the value of drugs prescribed is in crores but a significant proportion of drugs is prescribed irrationally. This is probably due to defect in prescription pattern, lack of knowledge in health care personnel, pressure from drug manufacturing companies and many more. To promote rational use of drugs, standard policies must be set and this can be achieved only after auditing current prescription practices.

Methods: A prospective study was carried out in Medical college hospital, Raigarh in the month of January 2018. Around 1000 prescriptions were collected randomly from pharmacy and the prescriptions were analysed on various parameters like patient's demography, parts of a prescription, information related to doctor and drugs.

Results: In this study, we found that percentage of generic drugs were 58.02\% and $70.43 \%$ drugs were from essential drug list. Only $13.19 \%$ FDCs were used. 34 prescriptions were illegible and capital letters were used only in 26 prescriptions. We also found deficiency in parts of a prescription like inscription part (13.3\%), subscription part (26.9\%) and in doctor's identity $(33.2 \%)$. The majority of drugs were antimicrobials $(23.81 \%)$ followed by anti-inflammatory and analgesics $(21.1 \%)$.

Conclusions: This study shows that the use of generic drugs and essential drugs is on the lower side as compared to standard guidelines. The prescription pattern was defective in many prescriptions. This study shows incompleteness of prescriptions and proper steps are needed to guide the physicians to promote rational use of drugs.
\end{abstract}

Keywords: Audit, Prescription, Polypharmacy, Rational

\section{INTRODUCTION}

According to WHO, drug utilization study can be defined as marketing, distribution, prescription and use of drugs in a society with special emphasis on resulting medical, social and economic consequences. According to WHO, rational use of drugs requires that patients receive medications appropriate to their clinical needs, in doses that meet their own individual requirements for an adequate period of time, at the lowest cost to them and their community. ${ }^{1}$

The importance of drug utilization studies can be magnified by connecting prescription data to the reasons for which drugs were prescribed. In the out patient department a prescription becomes the endpoint of a patient's visit to doctor most of the time. The prescription is the important instructive document from prescriber to a 
dispenser which should be written clearly, specifically and completely. ${ }^{2}$ Drugs play an important role in health care delivery system and treating disease. For comprehensive health care, we need availability and affordability of good quality medicines. Rational use of available drugs is needed for effective health care. In countries like India rational use of drugs is far below expectation and this is due to irrational prescribing and dispensing. ${ }^{3}$ World Health Organization states that more than half of all drugs are prescribed, dispensed or sold irrationally. ${ }^{4}$ Prescribing errors are the commonest form of avoidable medication errors. 5

For this purpose, WHO has published a number of indicators which include prescribing indicators, patient care indicators and the facility indicators. ${ }^{6}$ A number of studies have been carried out based on these indicators at various levels with the goal of studying the existing standards of medical care and scope for improvement. ${ }^{7-9}$ Drug utilization studies try to modify the prescribing patterns with the goal of making the medical care rational and cost effective. Thus, study of prescription patterns is needed to evaluate rational use of drugs and to magnify utilization of resources.

\section{METHODS}

The prospective study was carried out over a period of 6 working days in the month of January 2018 in LSLAM Government Medical College Hospital, Raigarh. Patients of either sex and age seeking care in various outpatient departments of Medical college hospital Raigarh were included in the study. Patients requiring admission in hospital for various reasons were excluded from the study. A total number of 1000 OPD prescriptions were collected from pharmacy department of Medical College Hospital. Prescriptions were collected randomly. Based on data in these prescriptions analysis was done on various parameters like

\section{Legibility of prescription}

It was assessed mainly by observing whether all parts of prescription were clear for reading with or without effort.

\section{Different parts of the prescription}

- Superscription including date, name, age, address, gender, weight, diagnosis mentioned.

- Inscription including name and strength of drugs.

- Subscription is the instruction to the pharmacist.

- Transcription: direction to the patients regarding the methods of administration.

- $\quad$ Signature and identification.

\section{WHO prescribing indicators}

- Average number of drugs per prescription-to measure polypharmacy.
- Percentage of drugs prescribed by generic names- to evaluate generic drug prescribing habits.

- Percentage of drugs prescribed from essential medicine list - to see whether drugs were prescribed according to national drug policy.

- Percentage of fixed dose combinations.

\section{RESULTS}

Total 1000 prescriptions were analyzed. 731 prescriptions were legible $(73.1 \%)$ which means they can be read comfortably without any help. $235(23.5 \%)$ were legible after additional effort of health personnel. 34(3.4\%) prescriptions were termed illegible which means handwriting of these 34 became difficult to read even with the help of health personnel. As a result, some of the calculations were based on 966 legible prescriptions (Figure 1).

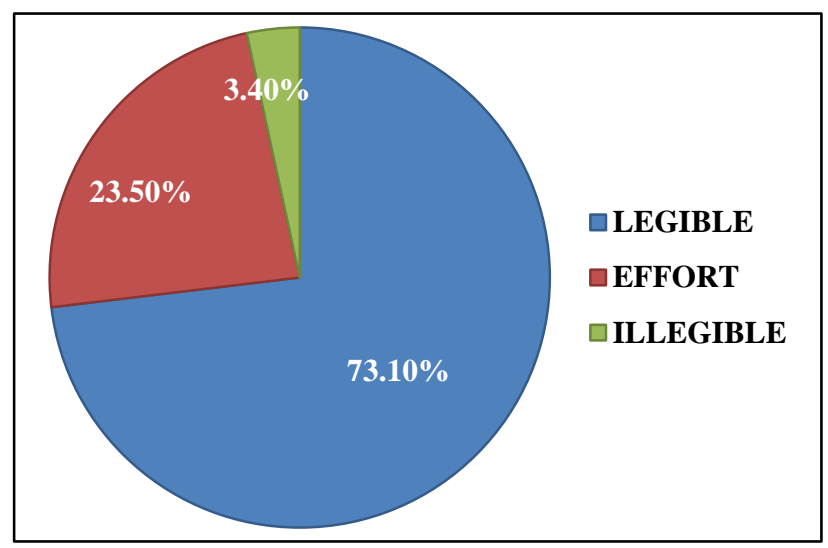

Figure 1: Legibility of prescriptions.

In the analysis of parts of a prescription, all 1000 prescriptions were having patient's identity number, date, name, age and sex. $52.4 \%$ patients were males and $47.6 \%$ were females. $24.6 \%$ were in <18-year age group, $62.2 \%$ were adults and rest $13.2 \%$ belong to geriatric ag group (Table 1).

Table 1: Demographic profile of patients.

\begin{tabular}{|ll|}
\hline Distribution & Percentage \\
\hline Age & \\
\hline$<18$-year age group & $24.6 \%(246)$ \\
\hline 19-65-year age group & $62.2 \%(622)$ \\
\hline$>65$-year age group & $13.2 \%(132)$ \\
\hline Sex & \\
\hline Male & $52.4 \%$ \\
\hline Female & $47.6 \%$ \\
\hline Transgender & - \\
\hline
\end{tabular}

In 133 legible prescriptions (13.76\% in total 966) name and strength of drugs were not written properly. The directions to pharmacist part was defective in 269 legible prescriptions $(28.73 \%)$. Doctor's signature was present in all prescriptions. In 33 prescriptions (3.41\%) doctor's 
registration number were missing. Doctor's designation/seal was absent in 332 prescriptions of total $966(34.36 \%)$. Names of the drugs were written in block letters in 26 prescriptions (2.69\% of total) (Table 2).

Table 2: Assessment of parts of prescription.

\begin{tabular}{|ll|}
\hline Prescription Parameters & $\begin{array}{l}\text { Percentage (in total } \\
966 \text { prescriptions) }\end{array}$ \\
\hline $\begin{array}{l}\text { Defective name and strength of } \\
\text { drug }\end{array}$ & $13.76 \%(133)$ \\
\hline Drugs written in block letters & $2.69 \%(26)$ \\
\hline Defective direction & $28.73 \%(269)$ \\
\hline $\begin{array}{l}\text { Absence of doctor's registration } \\
\text { number }\end{array}$ & $3.41 \%(33)$ \\
\hline $\begin{array}{l}\text { Absence of doctor's } \\
\text { designation/seal }\end{array}$ & $34.36 \%(332)$ \\
\hline
\end{tabular}

In 966 legible prescriptions, 3288 drugs were prescribed. 50 prescriptions were having one drug $(5.17 \%), 147$ prescriptions were having 2 drugs (15.21\%), 388 having 3 drugs (40.16\%), 201 prescriptions having 4 (20.80\%), 104 having 5 and 76 having 6 drugs (7.86\%) (Table 3 ).

Table 3: Percentage of number of drugs prescribed.

\begin{tabular}{|ll|}
\hline No. of drugs prescribed & $\%$ of prescription \\
\hline One drug & $5.17 \%$ \\
\hline Two drugs & $15.21 \%$ \\
\hline Three drugs & $40.16 \%$ \\
\hline Four drugs & $20.80 \%$ \\
\hline Five drugs & $10.76 \%$ \\
\hline Six drugs & $7.86 \%$ \\
\hline
\end{tabular}

In 3288 drugs, 1908 drugs (58.02\%) were prescribed by their generic names and 2316 drugs $(70.43 \%)$ were from the National essential drug list. 434 fixed dose combinations $(13.19 \%)$ were used in total 3288 drugs (Figure 2).

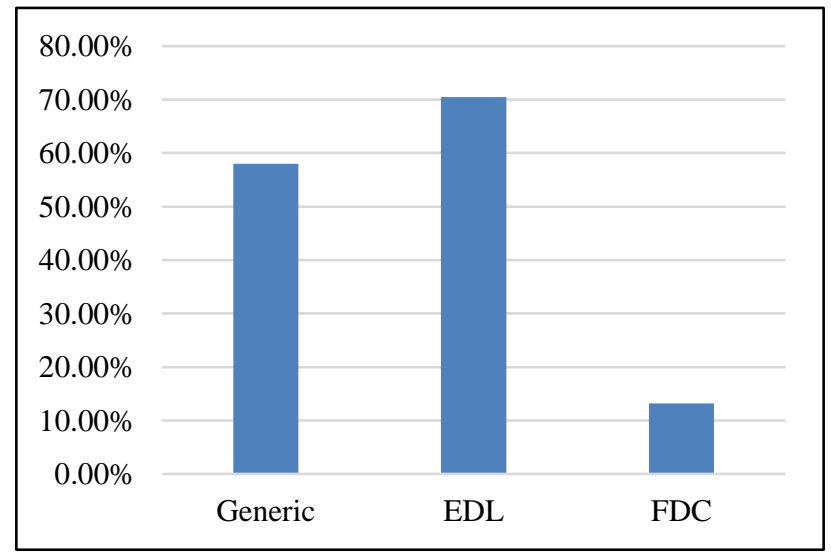

Figure 2: Percentage of generic drug, essential drug and FDC.

In 966 legible prescriptions, diagnosis was written in 808 prescriptions. One diagnosis was written in 679 prescriptions (70.29\% in 966), two in 106 (10.97\% in 966) and more than two in 23 prescriptions (2.38\%). No diagnosis was written in 158 prescriptions (16.36\% in 966) (Figure 3).

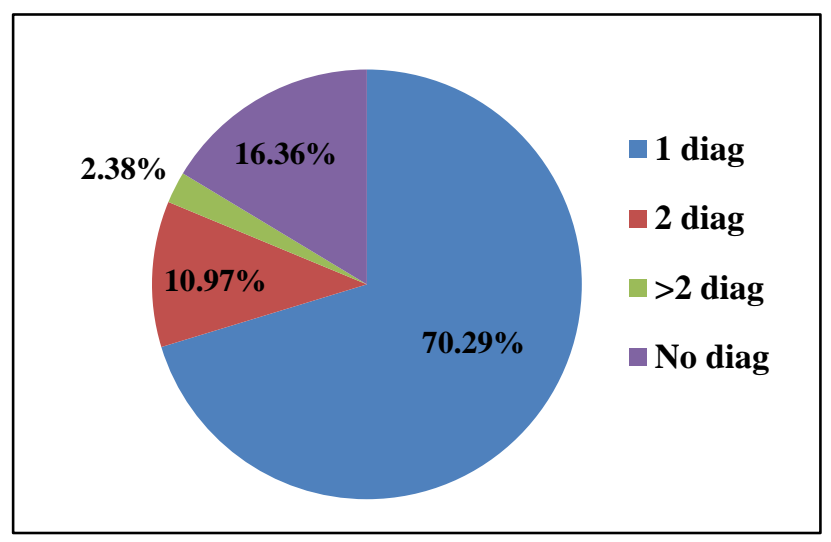

Figure 3: Number of diagnosis written in prescription (in percentage).

In total 3288 drugs, $84.09 \%$ drugs (2765) were in oral dosage form, 6.24\% (205) injections and 9.67\% (318) were topical agents (Figure 4).

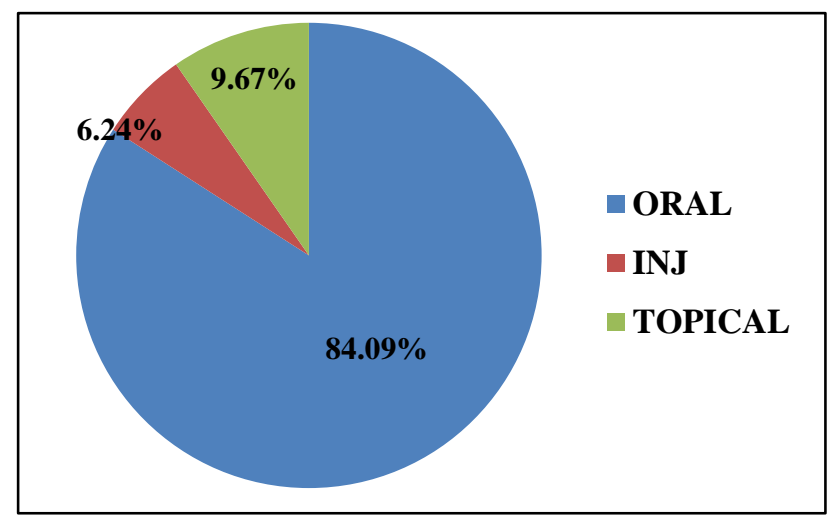

Figure 4: percentage of routes through which total drugs were prescribed.

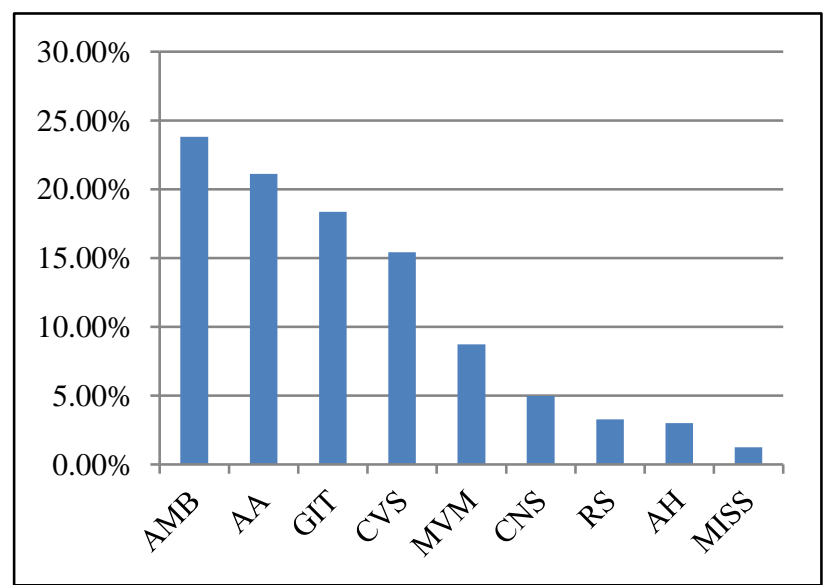

Figure 5: Distribution of prescribed drugs according to systems in text books of pharmacology. 
In 3288 drugs, 783 were antimicrobials (23.81\%), 695 drugs were analgesic and anti-inflammatory (21.13\%), 604 drugs $(18.36 \%)$ fall under GIT system, 507 cardiovascular drugs (15.41\%), 287 multivitamin and minerals $(8.72 \%)$, 164 CNS drugs $4.98 \%$ ), respiratory system drugs 108 (3.28\%), antihistamines $99(3.01 \%)$ and miscellaneous 41 $(1.24 \%)$ (Figure 5).

\section{DISCUSSION}

Rational use of drugs is an important tool of health care. Besides patient's well being it also has many other social and financial implications. It leads to the fair use of limited resources which is particularly helpful for a developing country like India. To assess the quality of a prescription the mainstay is prescription audit. The data obtained from the audit may act as a guide for the prescribers, policy makers and administrators.

In our study after screening 1000 prescriptions, we found deficiencies in various aspects. First of all, only names of 26 drugs were written in block letters which means that only $2.6 \%$ prescriptions were following the recent guidelines. Use of capital letters automatically increases the legibility of prescriptions. ${ }^{10-12}$

Around 58\% drugs were written in generic names. This is not satisfactory as compared to other studies. ${ }^{11}$ This indicates that our prescribing tendencies are typically influenced by the representatives of drug manufacturing companies for bidirectional profits. The cost per prescription also gets increased for this. The use of generic drugs reduces the incidence of dispensing error. ${ }^{12}$ The prescribers therefore must be aware of the use of generic drugs and for this regular meeting should be arranged to make them aware about the advantage of using generic drugs.

Fixed dose combinations were $13.19 \%$ which is quite satisfactory as compared to other studies. Most of them were antimicrobial agents. Use of fixed dose combinations should be discouraged unless strictly necessary. ${ }^{13-14}$

Among 3288 prescribed drugs $23.81 \%$ were antibiotics. As compared to other studies this figure is quite low and acceptable. But there were many patients having diagnosed of upper respiratory tract infections which are self-limiting. Antibiotic usage should be appropriate, otherwise drug resistance is inevitable. Culture sensitivity testing should be done prior to the use of antibiotics. ${ }^{15}$ There should be proper guidelines regarding antimicrobial drug prescribing, so that the clinicians can choose an antibiotic appropriate for specific patient's needs. ${ }^{15}$ The average number of drugs prescribed in 966 prescriptions were 3.4. This figure is not fit for declaring polypharmacy but when individual prescriptions were analyzed polypharmacy was clearly visible as 104 prescriptions were having 5 drugs and 76 were containing 6 drugs. Polypharmacy is the prime reason behind adverse drug reactions and drug interactions. It also increases financial burden on patients. Even multivitamins, minerals and herbal products are involved in drug interactions and adverse drug reactions. ${ }^{16}$

The most common categories of drugs prescribed to outpatients were antimicrobials $(23.81 \%)$ followed by analgesic and anti-inflammatory drugs (21.11\%). So, infection, musculoskeletal pain, fever and inflammation became the prime causes for which around $45 \%$ drugs were prescribed. In some prescriptions, where an analgesic like paracetamol may be sufficient was replaced by ibuprofen and diclofenac. A significant number of multivitamins and minerals were prescribed without any specific indication. Similar was case of antihistamines and cough syrups. The usage of these drugs without any specific indication may just increase the incidence of adverse effects.

\section{CONCLUSION}

Prescription audit is the current need to promote rational use of drugs, to prevent medication error and to decrease financial burden on patients. In our study, many parts of prescriptions were deficient, and it is necessary to educate medical personnel regarding standard prescription patterns. To improve the quality of prescriptions, doctors in the hospitals should be provided with standard treatment guidelines, list of Essential drugs and FDCs. Regular CME and workshops along with personal interaction with medical personnel will also help to improve the standards of prescription writing.

\section{Funding: No funding sources Conflict of interest: None declared \\ Ethical approval: The study was approved by the Institutional Ethics Committee}

\section{REFERENCES}

1. Hogerzeil HV. Promoting rational prescribing: an international perspective. $\mathrm{Br} \mathrm{J}$ Clin Pharmacol. 1995;39:1-6.

2. Meyer TA. Improving the quality of the order-writing process for inpatient orders and outpatient prescriptions. Ame J Health-System Pharmacy. 2000;57(Suppl.4):S18-2.

3. Karande S, Sankhe P, Kulkarni M. Patterns of prescription and drug dispensing. Ind $\mathrm{J}$ Pediatr. 2005;72(2):117-21.

4. Dean B, Schachter M, Vincent C, Barber N. Prescribing errors in hospital inpatients: their incidence and clinical significance. Qual Saf Health Care. 2002;11:340-4.

5. Hennessy S, Bilker WB, Zhou L, Weber AL, Brensinger $\mathrm{C}$, Wang $\mathrm{Y}$, et al. Retrospective drug utilization review, prescribing errors, and clinical outcomes. JAMA. 2003;290(11):1494-9.

6. World Health Organization. The Rational use of drugs: report of the conference of experts, Nairobi, 2529 November 1985. World Health Organization; 1987.

7. Debalto D, Gobe Z, Teklemariam S. A base line survey of prescribing indicators and factors 
influencing prescribing in southern Ethipia. J Health Dev. 1991;11(3):263-7.

8. Desta Z, Abula T, Gebre-Yohannes A, Worku A. Drug prescribing pattens for outpatients in three hospitals in north-west Ethiopia. Ethiop J Health Dev. 2002;16(2):183-9.

9. Hogerzeil HV, Walker GJA, Sallami AO, Fernando G. Impact of an essential drugs program on availability and rational use of drugs. Lancet. 1989;1(8630):1412.

10. Rodríguez-Vera FJ, Marin Y, Sanchez A, Borrachero C, Pujol E. Illegible handwriting in medical records. J Royal Soci Med. 2002;95(11):545-6.

11. Bruner A, Kasdan ML. Handwriting errors: harmful, wasteful and preventable. J Ky Med Assoc. 2001;99:189-92.

12. Winslow EH, Nestor VA, Davidoff SK, Thompson PG, Borum JC. Legibility and completeness of physicians' handwritten medication orders. Heart Lung. 1997;26(2):158-64.
13. Kastury N, Singh S, Ansari KU. An audit of prescription for rational use of fixed dose drug combinations. Ind J Pharmacol. 1999;31:367-9.

14. Chakrabarti A. Prescription of fixed dose combination drugs for diarrhea. Ind J Med Ethics 2007;4:165-7.

15. Gupta N, Sharma D, Garg SK, Bhargava VK. Auditing of prescriptions to study utilization of antimicrobials in a tertiary hospital. Ind J Pharmacol. 1997;29(6):411.

16. Patel PS, Rana DA, Suthar JV, Malhotra SD, Patel VJ. A study of potential adverse drug interactions among prescribed drugs in medicine outpatient department of a tertiary care teaching hospital. J Basic Clin Pharm. 2014;5:44 8.

Cite this article as: Raj P, Choudhury S, Kundu S, Patel S, Sidar B. Study of prescription pattern in a tertiary care hospital in Chhattisgarh, India: an observational study. Int J Basic Clin Pharmacol 2018;7:598-602. 